\title{
Detectability of Gravitational Waves from Phase Transitions
}

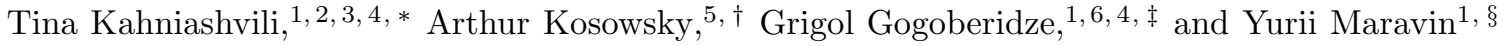 \\ ${ }^{1}$ Department of Physics, Kansas State University, 116 Cardwell Hall, Manhattan, KS 66506, USA \\ ${ }^{2}$ CCPP, New York University, 4 Washington Plaza, New York, NY 10003, USA \\ ${ }^{3}$ Department of Physics, Laurentian University, Ramsey Lake Road, Sudbury, ON P3E 2C6, Canada \\ ${ }^{4}$ National Abastumani Astrophysical Observatory, 2A Kazbegi Ave, Tbilisi, GE-0160, Georgia \\ ${ }^{5}$ Department of Physics and Astronomy, University of Pittsburgh, 3941 O'Hara Street, Pittsburgh, PA 15260 USA \\ ${ }^{6}$ Centre for Plasma Astrophysics, K.U. Leuven, Celestijnenlaan 200B, 3001 Leuven, Belgium
}

(Dated: October 27, 2018)

\begin{abstract}
Gravitational waves potentially represent our only direct probe of the universe when it was less than one second old. In particular, first-order phase transitions in the early universe can generate a stochastic background of gravitational waves which may be detectable today. We briefly summarize the physical sources of gravitational radiation from phase transitions and present semi-analytic expressions for the resulting gravitational wave spectra from three distinct realistic sources: bubble collisions, turbulent plasma motions, and inverse-cascade helical magnetohydrodynamic turbulence. Using phenomenological parameters to describe phase transition properties, we determine the region of parameter space for which gravitational waves can be detected by the proposed Laser Interferometer Space Antenna. The electroweak phase transition is detectable for a wide range of parameters.
\end{abstract}

PACS numbers: $98.70 . V c, 98.80 .-\mathrm{k}$

\section{INTRODUCTION}

Since gravitational waves propagate freely through the universe after being generated, their detection provides a powerful test of the very early universe. Various mechanisms that generate such gravitational waves have been discussed: quantum fluctuations during [1] and shortly after inflation [2]; bubble wall motion and collisions during phase transitions [3, 4, [5, 6, 7]; cosmic strings and defects, including primordial black holes [8]; cosmological magnetic fields [9, 10, 11], and plasma turbulence [12, 13, 14, 15]. This paper focuses on gravitational waves generated during phase transitions and study the possibility of direct detection by the planned Laser Interferometer Space Antenna (LISA),

Near-future data from the Large Hadron Collider will for the first time have the ability to probe in detail physics at the electroweak energy scale. This physics determines the nature of the electroweak phase transition in the early universe, when the primordial plasma went from an electroweak-symmetric state to a broken state with distinct electromagnetic and weak interactions. The phase transition took place when the primordial plasma had a temperature on the order of $1 \mathrm{TeV}$. Intriguingly, the Hubble frequency $H_{*}$ at this epoch, redshifted to today, falls in the lower end of the detection range for LISA, which ranges from $10^{-4}$ to $10^{-1} \mathrm{~Hz}$ [16]. If gravitational waves are generated during the electroweak phase transition, their characteristic frequency will be related to this

\footnotetext{
*Electronic address: tinatin@phys.ksu.edu

${ }^{\dagger}$ Electronic address: kosowsky@pitt.edu

‡Electronic address: gogober@geo.net.ge

$\S$ Electronic address: maravin@phys.ksu.edu
}

Hubble frequency: remarkably, gravitational waves may provide us with an alternate route to probing electroweak physics.

Having gravitational waves at detectable frequencies is not sufficient for detecting the electroweak phase transitions: the source of gravitational waves must be sufficiently strong to produce radiation with a detectably large amplitude today [17, 18, 19, 20]. If the phase transition is first order, the latent heat of the phase transition partly is transferred into kinetic energy of the walls of expanding bubbles of the broken phase. If these expanding bubbles contain a large enough amount of energy, they will produce a gravitational wave background with a detectably large amplitude today [3]. Models of the electroweak phase transition based on standard-model particle physics do not produce observable gravitational wave signals since they are not first order for allowed values of the Higgs mass [21], but common extensions of the standard model, including supersymmetry and extra dimensions, can produce much stronger phase transitions 22].

Bubbles of broken phase in a first-order phase transition expand and percolate to convert the entire universe to the low-energy phase. The kinetic energy in the bubble walls eventually thermalizes, but prior to that the bubbles act to stir the primordial plasma, plausibly generating Kolmogoroff turbulence cascading from the turbulence scale to a much smaller scale where the kinetic energy turns into thermal energy via viscous heating. The turbulent eddy motions can also be a potent source of gravitational waves if the energy input is large enough 12. If a small magnetic seed field with nonzero helicity is generated at the phase transition [23, 24], magnetohydrodynamic effects can generate an inverse cascade, transferring energy to scales substantially larger than the stirring scale [15] and resulting in a detectably large sig- 
nal [25], and additionally can give the radiation a nonzero circular polarization [14] which is also directly detectable 26]. So gravitational wave generation from an early universe phase transition can be decomposed into three distinct sources: expanding bubbles of the broken phase, hydrodynamic turbulence stirred by the colliding bubbles, and an inverse cascade due to the amplification of seed magnetic fields.

Previous papers have computed the gravitational wave spectra for each of these sources individually. Here we consider their combined spectrum. The detailed shape and amplitude of the spectrum depends on fundamental properties of the phase transition: its energy scale, bubble nucleation rate, latent heat, efficiency of converting latent heat into plasma motions, and mean helicity of seed magnetic fields. We summarize how the total power spectrum depends on these parameters, then evaluate the regions of parameter space for which the relic gravitational radiation from the phase transition is detectable with LISA, displaying the results as contour plots in the parameter space. While the bubble collisions produce detectable gravitational radiation for a very limited range of parameters, adding the radiation from turbulence and an MHD inverse cascade greatly widens the range of detectable phase transitions.

In the following Section, we give a brief overview of the physics of phase transitions and the corresponding phenomenological parameters. In Sec. III, we express the gravitational wave spectra in terms of these phenomenological parameters, and show how the total gravity wave spectrum varies with the parameters. Sec. IV compares these spectra with the projected LISA sensitivity curve for stochastic backgrounds, displaying detectability regions in the space of phase transition parameters.

\section{MODELING GRAVITATIONAL RADIATION}

\section{A. General Remarks on Gravitational Waves Generation}

Gravitational waves generated during phases transitions by stochastic sources have an energy density just after the phase transition of [27]

$$
\begin{aligned}
& \rho_{\mathrm{GW}}(\mathbf{x})=\frac{1}{32 \pi G}\left\langle\partial_{t} h_{i j}(\mathbf{x}, t) \partial_{t} h_{i j}(\mathbf{x}, t)\right\rangle= \\
& =\frac{G}{2 \pi} \int \mathrm{d}^{3} \mathbf{x}^{\prime} \mathrm{d}^{3} \mathbf{x}^{\prime \prime} \frac{\left\langle\partial_{t} S_{i j}\left(\mathbf{x}^{\prime}, t^{\prime}\right) \partial_{t} S_{i j}\left(\mathbf{x}^{\prime \prime}, t^{\prime \prime}\right)\right\rangle}{\left|\mathbf{x}-\mathbf{x}^{\prime}\right|\left|\mathbf{x}-\mathbf{x}^{\prime \prime}\right|} .
\end{aligned}
$$

where time variables with primes represent the lightcone combination $t^{\prime}=t-\left|\mathbf{x}-\mathbf{x}^{\prime}\right|, i$ and $j$ are spatial indices (repeated indices are summed), the source $S_{i j}(\mathbf{x}, t)=T_{i j}(\mathbf{x}, t)-\delta_{i j} T_{k}^{k}(\mathbf{x}, t) / 3$ is the traceless part of the stress-energy tensor $T_{i j}, G$ is the gravitational constant, and we use natural units with $\hbar=c=1$. This expression assumes that the duration of the phase transition is short enough to neglect the cosmological expansion during gravitational wave production. Since the phase transition lasts for a finite duration, we can consider the total radiation field to be an incoherent sum of radiation from many sources, each with a size corresponding to the light travel distance during the phase transition. With this approximation, we can apply the far-field approximation for each individual source region, $x \gg d$ where $d$ is the size of the source region; in this region the gravitational waves are the only metric perturbations 28], allowing replacement of $\left|\mathbf{x}-\mathbf{x}^{\prime}\right|$ by $|\mathbf{x}|$ in Eq. (1).

Generated gravitational waves propagate freely through the expanding universe until today. Their wavelengths simply scale with the scale factor $a$ of the universe, while their total energy density evolves like $a^{-4}$ and their amplitude decays like $a^{-1}$. Following Ref. [27], we use the gravitational wave spectral energy density $\rho_{G W}(\omega)$ per logarithmic angular frequency $\omega$, defined as the root-mean-square average of $\rho_{G W}(\mathbf{x}, \omega)$ over spatial positions, and form the spectral energy density parameter $\Omega_{G W}(\omega)=\rho_{G W}(\omega) / \rho_{c}$ with the critical energy density $\rho_{c}=3 H_{0}^{2} / 8 \pi G$. Changing to linear frequency $f=\omega / 2 \pi$, a characteristic strain amplitude is conventionally defined as

$$
h_{c}(f)=1.3 \times 10^{-18}\left(\frac{1 \mathrm{~Hz}}{f}\right)\left[h_{0}^{2} \Omega_{\mathrm{GW}}(f)\right]^{1 / 2}
$$

where $h_{0}$ is the current Hubble parameter $H_{0}$ in units of $100 \mathrm{~km} \mathrm{sec}^{-1} \mathrm{Mpc}^{-1}$. We will present our results in terms of the gravitational wave amplitude $h_{c}$ vs. linear frequency $f$ measured today.

The temperature at which the phase transition occurs determines the corresponding Hubble frequency, which is given today by [12]

$$
f_{\mathrm{H}}=1.6 \times 10^{-5} \mathrm{~Hz}\left(\frac{T_{*}}{100 \mathrm{GeV}}\right)\left(\frac{g_{*}}{100}\right)^{1 / 6}
$$

where $g_{*}$ is the number of relativistic degrees of freedom at the temperature $T_{*}$; for standard model degrees of freedom, $g_{*} \sim 106.75$ as $T \rightarrow \infty$. To obtain this expression, begin with the Hubble parameter at temperature $T_{*}$, given by

$$
H_{*}^{2}=\frac{8 \pi^{3} T_{*}^{4}}{90 M_{\mathrm{Pl}}^{2}}
$$

then rescale $H_{*}$ to its present value with the ratio between the scale factors $a_{*}$ corresponding to the Universe temperature $T_{*}$ and $a_{0}$ today:

$$
\frac{a_{*}}{a_{0}}=8 \times 10^{-16}\left(\frac{100 \mathrm{GeV}}{T_{*}}\right)\left(\frac{100}{g_{*}}\right)^{\frac{1}{3}} .
$$

Note that an extra factor of approximately 3.6 occurs for $f_{\mathrm{H}}$ in Ref. [29] (Eq. 10) due to approximating $H_{*}^{2} \simeq$ $G \rho_{\text {tot }}$.

It can be shown from general physical principles that since the gravitational wave generation process is causal, 
the peak frequency must be greater than or equal to the Hubble frequency, $f_{\text {peak }} \geq f_{\mathrm{H}}$ [3]. Independent of the nature of the source, the gravitational wave spectrum is the same at low frequencies, $\Omega_{\mathrm{GW}}(f) \propto\left(f / f_{\text {peak }}\right)^{3}$ (for $\left.f \ll f_{\text {peak }}\right)[5,11,12,15,18,30]$. The gravitational spectrum behavior at higher frequencies is determined by the specific source features.

\section{B. Phase Transition Model Parameters}

A first-order phase transition is generically described by several parameters: (i) $\alpha \equiv \rho_{\text {vac }} / \rho_{\text {thermal }}$, the ratio of the vacuum energy associated with the phase transition to the thermal density of the Universe at the time (which characterizes the strength of the phase transition); (ii) $\kappa$, an efficiency factor which gives the fraction of the available vacuum energy which goes into the kinetic energy of the expanding bubble walls, as opposed to thermal energy; (iii) $\beta^{-1}$, which sets the characteristic time scale for the phase transition; (iv) $v_{b}$, the velocity of the expanding bubble walls, which set the characteristic length scale of the phase transition; (v) $T_{*}$, the temperature at which the phase transition occurs. In any first-order phase transition, the characteristic bubble nucleation rate per unit volume is generically [31]

$$
\Gamma=\Gamma_{0} e^{\beta t},
$$

which uses the constant and linear terms of a Taylor expansion of the bubble nucleation action. General considerations confirmed by numerical calculations show that the largest bubbles reach a size of order $\beta^{-1} v_{b}$ by the end of the phase transition [6], where $v_{b}$ is the bubble expansion velocity, assuming the bubbles remain spherical as they expand. In general, $\beta$ is expected to be of the order $4 \ln \left(m_{\mathrm{Pl}} / T\right) H \simeq 100 H$ for a Hubble rate $H$ [31].

The fundamental symmetry breaking mechanism which drives the phase transition determines some effective potential for bubble nucleation [32]. The difference in energy density between the two phases and the bubble nucleation rate are both determined by this mechanism. Thus the parameters $T_{*}, \beta$, and $\alpha$ are all determined directly by the underlying physics. On the other hand, the bubble velocity $v_{b}$ and the fraction of kinetic energy into the bubbles $\kappa$ depend on the detailed microphysics involved in the bubble propagation through the relativistic plasma and are not determined from general properties of the effective potential. In general, the larger the vacuum energy density driving phase transition, the higher the bubble wall velocity $v_{b}[\underline{4}, 31]$.

\section{SOURCES OF GRAVITATIONAL RADIATION}

\section{A. Bubble collisions}

Bubbles walls can propagate outwards through a relativistic plasma via two modes, detonation and deflagration [33]. In the case of detonation, the bubble walls are thin compared to the bubble radius with velocity is 33]

$$
v_{b}(\alpha)=\frac{1 / \sqrt{3}+\left(\alpha^{2}+2 \alpha / 3\right)^{1 / 2}}{1+\alpha}
$$

and an approximate form of $\kappa$ is given [4]

$$
\kappa(\alpha)=\frac{1}{1+A \alpha}\left[A \alpha+\frac{4}{27}\left(\frac{3 \alpha}{2}\right)^{1 / 2}\right]
$$

with $A=0.715$. If the bubbles propagate as a deflagration front, the walls are thick and have lower energy density. However, it has been argued [34] that for relativistic plasma, instabilities in the bubble shape will accelerate the bubble walls and the hydrodynamic deflagration expansion mode is unstable to becoming a detonation. For this reason, Ref. [4] assumed that Eqs. (7) and (8) hold, and we will also make that assumption here. (For unusual cases with very strong detonation and $\alpha \gtrsim 1$, the following formulas must be corrected.) Both modes of bubble propagation recently have been re-addressed in Ref. [30], with results slightly different from those of Ref. [4].

The peak frequency of the gravitational wave spectrum is of fundamental importance for issues of detectability. The bubble nucleation time scale is always determined by $\beta^{-1}$, while the largest bubble size depends also on the bubble wall velocity, $l_{0}=v_{b} \beta^{-1}$. What scale determines the peak frequency of the gravitational waves? When the bubble wall velocity $v_{b}$ approaches the speed of light, $l_{0}^{-1} \simeq \beta$, and both characteristic frequency scales are the same. In Refs. [3, 4, [5], where the bubble wall velocity is assumed to be the speed of light, the peak frequency is given by [4, 18, 30]

$$
f_{\text {peak }}^{\mathrm{BC}}=0.3\left(\frac{\beta}{H_{\star}}\right) f_{\mathrm{H}} .
$$

Note the angular peak frequency at the moment of emission $\omega_{\text {peak } \star}^{\mathrm{BC}}$ is set by $0.3 k_{0} v_{b} \simeq 2 \beta$ (where $\left.k_{0}=2 \pi / l_{0}\right)$. To understand the peak frequency of the radiation from bubble walls with lower velocity, it is useful to exploit the direct analogy with sound wave generation. The typical source velocity compared to the velocity of the induced waves determines the characteristic peak frequency [35]. If the characteristic source velocity, given by the ratio between the source's characteristic length and time scales, is less than the group velocity of the induced waves, then the frequency is determined by the source time scale; in the opposite case, the characteristic length scale determines the peak frequency. Applied to gravitational 
waves with group velocity $v=1$, the source time scale always determines the peak radiation frequency, since by causality its characteristic velocity can never exceed the speed of light.

For the realistic values $\beta / H_{*}=100$ and $g_{*}=100$, the peak frequency from bubble collisions $f_{\text {peak }}^{\mathrm{BC}} \simeq 5 \times 10^{-4} \mathrm{~Hz}$ [18] is close to the peak of LISA sensitivity at $2 \mathrm{mHz}$ if $T_{*} \simeq 100 \mathrm{GeV}$. The corresponding peak amplitude of the gravitational radiation today is [4]

$$
\begin{aligned}
h_{c}\left(f_{\text {peak }}^{\mathrm{BC}}\right) & =1.8 \times 10^{-16} \kappa\left(\frac{\alpha}{\alpha+1}\right)\left(\frac{H_{\star}}{\beta}\right)^{2}\left(\frac{100}{g_{\star}}\right)^{\frac{1}{3}} \\
& \times\left(\frac{100 \mathrm{Gev}}{T_{\star}}\right)\left(\frac{v_{b}^{5}}{v_{b}^{3}+0.24}\right)^{\frac{1}{2}} .
\end{aligned}
$$

The spectrum shape at high frequencies $\left(f \gg f_{\mathrm{H}}\right)$ is $h_{c}(f) \propto f^{-2}[30]$, very close to the scaling $h_{c}(f) \propto f^{-1.9}$ found numerically in Ref. [4].

\section{B. Turbulence}

Once the bubbles expand and percolate, a significant amount of their kinetic energy will be converted to turbulent bulk motions of the primordial plasma [5]. As the phase transition ends, far more small bubbles have been nucleated than large ones, but the energy density in the large ones dominates the total energy density [31]. Therefore, a reasonable approximation is that the turbulence energy is injected on a stirring scale $l_{0}$ corresponding to the size of the largest bubbles, and that that the stirring will last for roughly $\tau_{\text {stir }}=\beta^{-1}$, comparable to the duration of the phase transition. We make the conservative assumption that the duration of the turbulence is also $\tau_{\text {stir }}$. The turbulence can actually last substantially longer than this; see Ref. [12] for a detailed discussion. After generation, the turbulence kinetic energy cascades from larger to smaller scales, stopping at a viscous damping scale $l_{D}$ where the turbulence energy is dissipated into heat. We define the parameter $\gamma \equiv l_{0} H_{\star} \simeq v_{b}\left(H_{*} / \beta\right)$ which determines the number $\left(N_{\text {eddy }} \simeq \gamma^{-3}\right)$ of turbulent eddies within the Hubble radius. In terms of the parameter $\gamma$, Eq. (9) reads $f_{\text {peak }}^{\mathrm{BC}}=0.34 f_{\mathrm{H}} v_{b} / \gamma$.

We associate a characteristic velocity perturbation $v_{0}$ with the fluid velocity of the largest eddies at the stirring scale $l_{0}$. This velocity determines the turbulent motions' Mach number $M \simeq v_{0}$. It is easy to estimate $v_{0}$ through relating the kinetic energy density of plasma $\mathrm{w} v_{0}^{2} /\left(1-v_{0}^{2}\right)$ (where $\mathrm{w}=p+\rho$ is enthalpy of plasma) and the vacuum energy $\rho_{\text {vac }}$ using $\mathrm{w}=4 \alpha \rho_{\mathrm{vac}} / 3$. This leads to [18]

$$
v_{0}=\sqrt{\frac{3 \kappa \alpha}{4+3 \kappa \alpha}} .
$$

Accounting for Eq. (8), $v_{0}$ can be expressed uniquely in terms of $\alpha$ and in the case of realistic phase transitions, when $\alpha<1, v_{0} \simeq \alpha^{3 / 4}(3 / 2)^{1 / 4} / 3$, and of course is less than the bubble wall expansion speed $v_{b}$. For $\kappa \alpha \simeq 1$ corresponding to a strongly first order phase transition, $v_{0} \simeq 0.65$.

Figure 1 of Ref. [15] shows that it is safe to adopt the aero-acoustic limit $k \rightarrow 0$ in the gravitational wave generation formalism for turbulent sources, provided only that the Mach number $M \lesssim 1$. Employing this simplifying assumption and adopting the results of Ref. [15], the peak frequency of gravitational waves is given by

$$
f_{\text {peak }}^{\mathrm{T}}=\left(\frac{M}{v_{b}}\right) f_{\mathrm{H}}\left(\frac{\beta}{H_{\star}}\right) \simeq 0.3\left(\frac{M}{v_{b}}\right) f_{\text {peak }}^{\mathrm{BC}} .
$$

For typical parameters $\beta=100 H_{*}, T_{*}=100 \mathrm{GeV}, g_{*}=$ 100 , and $\alpha=0.5$ (which correspond to $\kappa=0.36, v_{b}=$ 0.89 and $v_{0}=0.34$ ), the peak frequency is $0.4 \mathrm{mHz}$, within the LISA range.

At frequencies high compared to the characteristic damping frequency, $f \gg f_{D} \equiv f_{\text {peak }}^{\mathrm{T}} R^{1 / 2}$ with $R$ is the turbulence Reynolds number, the gravitational wave strain spectrum $h_{c}(f) \sim\left(f / f_{D}\right)^{1 / 2} \exp \left(-\left(f / f_{D}\right)^{2}\right)$ possesses a sharp exponential cutoff. Near the peak frequency, for $f_{\text {peak }}^{\mathrm{T}}<f<f_{D}$, the spectrum scales like $h_{c}(f) \simeq\left(f / f_{\text {peak }}^{\mathrm{T}}\right)^{-13 / 4}[15]$. The peak frequency of the radiation spectrum from bubble collisions for the parameters considered here is lower than the peak frequency due to turbulence, $f_{\text {peak }}^{\mathrm{BC}}<f_{\text {peak }}^{\mathrm{T}}$, and the bubble collision spectrum falls only as $\left(f / f_{\text {peak }}^{\text {BC }}\right)^{-2}$ at frequencies above the peak, but even at frequencies substantially greater than $f_{\text {peak }}^{T}$, the total gravitational wave spectrum is not dominated by the bubble collision source, due to the lower amplitude of gravitational waves induced by collisions.

The gravitational wave amplitude from turbulence can be conveniently estimated analytically using the aeroacoustic approximation (see Refs. [15, 25] for details of numerical computations), giving

$$
\begin{aligned}
h_{c}\left(f_{\text {peak }}^{\mathrm{T}}\right) & =3.3 \times 10^{-16} M^{\frac{3}{2}} v_{b}^{2}\left(\frac{H_{\star}}{\beta}\right)^{2}\left(\frac{100}{g_{\star}}\right)^{\frac{1}{3}} \\
& \times\left(\frac{100 \mathrm{GeV}}{T_{\star}}\right) \simeq 1.9 M^{\frac{3}{2}}\left(\frac{\alpha+1}{\kappa \alpha}\right) \\
& \times\left(\frac{v_{b}}{v_{b}^{3}+0.24}\right)^{-\frac{1}{2}} h_{c}\left(f_{\text {peak }}^{\text {BC }}\right)
\end{aligned}
$$

and for $\alpha \simeq 0.5$ is approximately 3 times larger that the peak amplitude coming from bubble collisions.

\section{MHD Inverse Cascade Turbulence}

Several models lead to generation of a cosmological helical magnetic field during phase transitions [23, 24]. In such a case, turbulence develops in a magnetized plasma, which is qualitatively different than an unmagnetized plamsa. In particular, an inverse cascade which generates magnetized perturbations on larger scales than the stirring scale can occur. To model such turbulence, we 
assume that the hydrodynamic and magnetic Reynolds numbers are much greater than unity on scales $\sim l_{0}$ (which is simply the condition for turbulence to develop); we also assmue that the dynamics of magnetohydrodynamic turbulence is dominated by Alfvén waves for which the magnetic and kinetic energy densities are in approximate equipartition [36]. We also assume a small initial magnetic helicity, $\zeta_{*} \equiv \mathcal{H}_{M}\left(t_{*}\right) /\left[2 \xi_{M}\left(t_{*}\right) \mathcal{E}_{M}\left(t_{*}\right)\right] \ll 1$, of a magnetic field with the magnetic-eddy correlation length $\xi_{M}(t) \equiv \int E^{M}(k, t) k^{-1} d k / \mathcal{E}_{M}(t)$ and with total magnetic energy $\mathcal{E}_{M}(t)=\int E^{M}(k, t) d k$ and helicity $\mathcal{H}_{M}(t)=\int H^{M}(k, t) d k$. From the stirring scale, the direct cascade proceeds via a Kolmogoroff-like model [37, 38] and the following inverse cascade stage adopts the MHD turbulence model of Refs. [39, 40]. Then it can be shown that the induced gravitational wave spectrum always peaks at the Hubble frequency $f_{\text {peak }}^{\mathrm{MHD}}=f_{\mathrm{H}}$ [25], and compared to the turbulence peak frequency it is shifted to lower frequencies by a factor $\left(v_{b} / M\right)\left(H_{*} / \beta\right)$.

The amplitude is larger compared to hydrodynamic turbulence case as [25]

$$
\begin{aligned}
h_{c}\left(f_{\text {peak }}^{\mathrm{MHD}}\right) & \simeq 32 \times \zeta_{*}^{9 / 8}\left(\frac{\gamma}{0.01}\right)^{-3 / 4} M^{3 / 4} h_{c}\left(f_{\text {peak }}^{\mathrm{T}}\right) \\
& =\left(\frac{M}{v_{b}}\right)^{3 / 4} \zeta_{*}^{9 / 8} h_{c}\left(f_{\text {peak }}^{\mathrm{T}}\right)
\end{aligned}
$$

This increase in amplitude is because the duration time of the inverse cascade is longer than the duration of the direct cascade. No efficient dissipation mechanisms exist at the largest scales, so the cascade will stop at a scale $\xi_{M}=1 / k_{S}$ either when the cascade time scale $\tau_{\text {cas }}$ reaches the expansion time scale $H_{*}^{-1}$ or when the characteristic length scale $\xi_{M}(t)$ reaches the Hubble length $H_{*}^{-1}$. These conditions are $\zeta_{*}^{-1 / 2} l_{S}^{2} / v_{0} l_{0} \leq H_{*}^{-1}$ or $l_{S}=2 \pi / k_{S} \leq H_{*}^{-1}$ (the cascade time is scale dependent and maximal at $k=k_{S}$ ). Defining $\gamma \leq 1$, it is easy to see that the first condition is fulfilled first, and consequently $k_{0} / k_{S} \leq\left(v_{0} / \gamma\right)^{1 / 2} \zeta_{*}^{1 / 4}$. To have an inverse cascade requires $k_{0} / k_{S} \geq 1$, leading to a constraint on initial helicity $\gamma \leq M \zeta_{*}^{1 / 2}$. In addition, these gravitational waves will be circularly polarized since they are induced by parity-violating stochastic sources [14]. If alternately we use the inverse cascade model of Ref. [41], the peak frequency of the gravitational wave spectrum remains unchanged while the peak amplitude is doubled [25, 42].

\section{Total Gravitational Wave Spectrum}

The total gravitational wave background from a firstorder phase transition is a sum of three terms corresponding to the three distinct sources discussed here. Our results are expressed in terms of the effective strain spectrum $h_{c}(f)$ in terms of the phase transition temperature $T_{*}$, the number of effective relativistic degrees of freedom

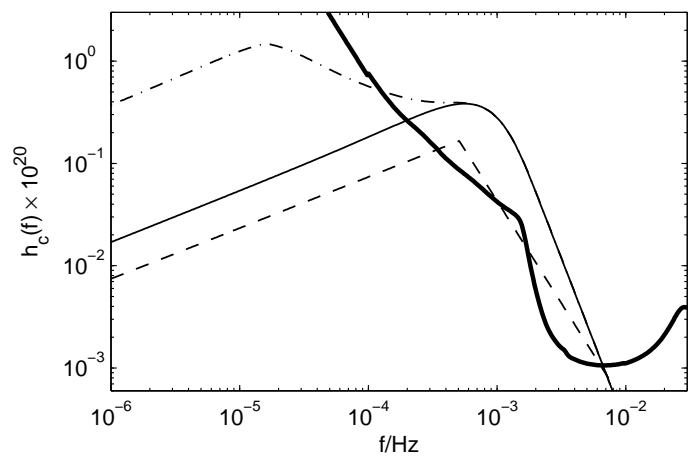

FIG. 1: The spectrum of gravitational radiation for a firstorder phase transition with $g_{*}=100, T_{*}=100 \mathrm{GeV}, \alpha=0.5$ and $\beta=100 H_{\star}$, from bubble collisions (dash line), hydrodynamic turbulence with zero helicity (solid line) and MHD turbulence with $\zeta_{*}=0.15$ (dash-dotted line). The bold solid line corresponds to the 1-year, $5 \sigma$ LISA sensitivity curve 43], including the confusion noise from white dwarf binaries [44].

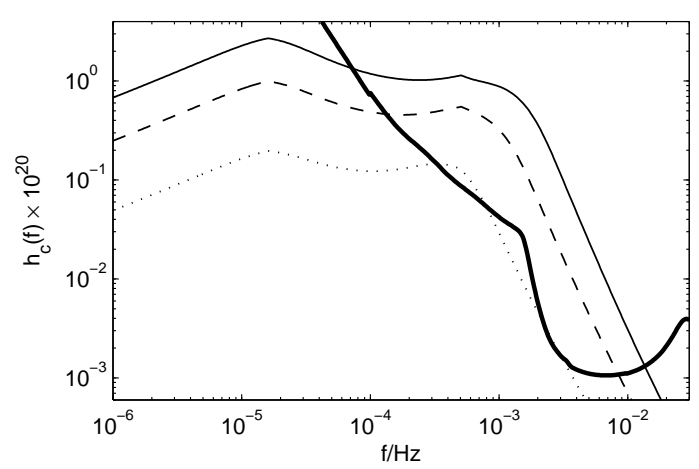

FIG. 2: The total spectrum of gravitational radiation (including there sources of gravitational radiation bubble collisions, hydro-turbulence, and MHD turbulence) for $g_{*}=100$, $T_{*}=100 \mathrm{GeV}, \zeta_{*}=0.1, \beta=100 H_{*}$, and three different values of $\alpha$ : $\alpha=1$ (solid line), $\alpha=0.5$ (dash line) and $\alpha=0.2$ (dotted line), with the LISA sensitivity curve..

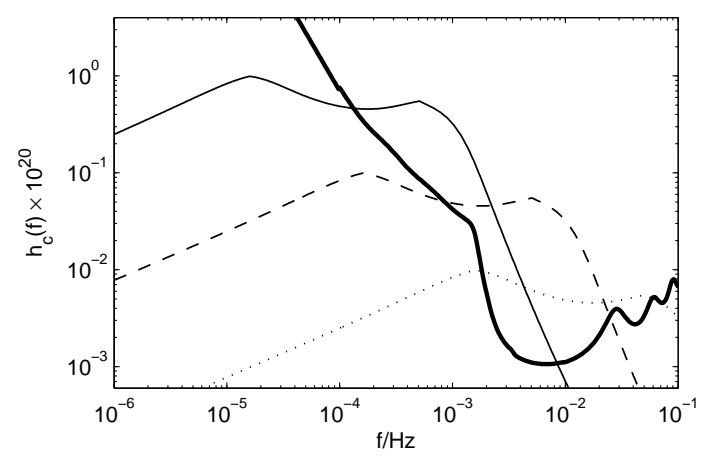

FIG. 3: The total spectrum of gravitational radiation for $g_{*}=100, \alpha=0.5, \beta=100 H_{*}, \zeta_{*}=0.1$, and three different temperature values, $T_{*}=100 \mathrm{GeV}$ (solid line), $T_{*}=1 \mathrm{TeV}$ (dash line), and $T_{*}=10 \mathrm{TeV}$ (dotted line), with the LISA sensitivity curve. 


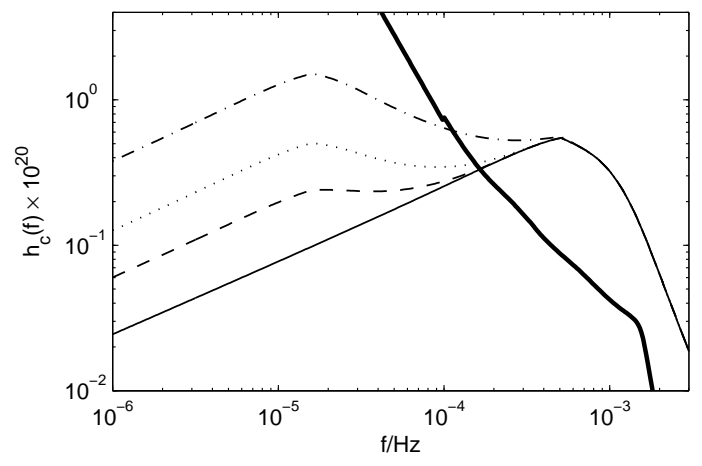

FIG. 4: The total spectrum of gravitational radiation for $g_{*}=100, T_{*}=100 \mathrm{GeV}, \alpha_{*}=0.5, \beta=100 H_{*}$, and four different values of $\zeta_{*}: \zeta_{*}=0$, corresponding to hydrodynamic turbulence without the inverse cascade effect (solid line), $\zeta_{*}=0.02$ (dash line), $\zeta_{*}=0.5$ (dotted line), and $\zeta_{*}=0.15$ (dot-dash line), with the LISA sensitivity curve.

$g_{*}$, the ratio of vacuum energy to thermal energy $\alpha$, the bubble nucleation time scale $\beta$, and the initial magnetic helicity parameter $\zeta_{*}$. All others relevant parameters appearing in the analytical approximations, such as $\kappa, v_{b}$, $l_{0}$, and $M$ can be computed in terms of these fundamental parameters using simple and well-motivated assumptions. We fix $g_{*}=100$, and we do not study the dependence on this parameter since it is very weak and the actual value of $g_{*}$ will not vary more than a factor of a few from this nominal value $\left(g_{*}=106.75\right.$ for standard model degrees of freedom at temperatures large compared to the $\mathrm{W}$ and top quark masses). We choose $\zeta_{*}$ small enough to insure that the resulting magnetic turbulence can be modeled as an inverse cascade modeling (see Ref. [25] for details).

Figure 1 displays the three components contributing to the total gravitational wave spectrum, for a fiducial model with $g_{*}=100, \alpha=0.5, \beta / H_{*}=100$, and $T_{*}=100$ $\mathrm{GeV}$. Bubble collisions are shown as the dashed line, hydrodynamic turbulence with no magnetic helicity as the solid line, and MHD turbulence with an initial helicity parameter $\zeta_{*}=0.15$. The dark curve is the LISA design sensitivity curve for stochastic backgrounds with a detection signal-to-noise ratio of 5 over a 1 -year integration [43], including a rough estimate of the confusion limit from white-dwarf binaries [44]. It is clear that bubble collisions alone do not produce a significant signal (marginally detectable) in LISA for this range of phase transitions parameters, agreeing with previous results [17, 18, 19, 30]. The turbulence peak is observable, as well as the separate peak from the MHD inverse cascade.

The next set of figures show how the total gravitational wave spectrum varies as each of the phase transition parameters is changed, holding the others fixed. Figure 2 shows the variation with $\alpha$, Fig. 3 the variation with $T_{*}$, Fig. 4 the variation with $\zeta_{*}$, and Fig. [5 the variation with $\beta / H_{\star}$. In each case, the spectrum for the fiducial model

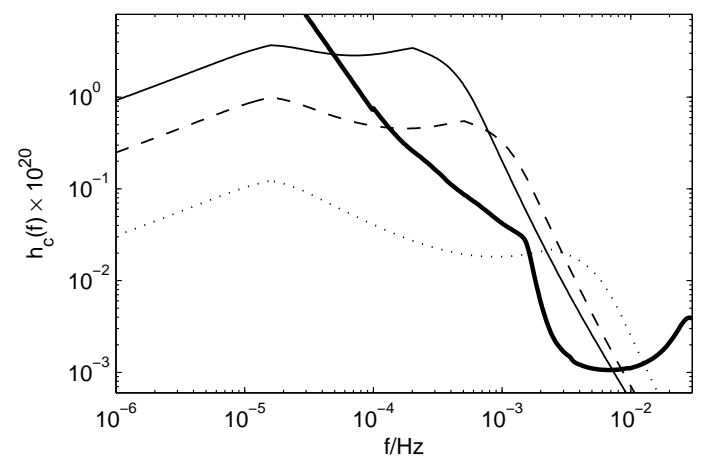

FIG. 5: The total spectrum of gravitational radiation for $g_{*}=$ $100, T_{*}=100 \mathrm{GeV}, \zeta_{*}=0.1, \alpha=0.5$, and three different values of $\beta$ : $\beta=40 H_{*}$ (solid line), $\beta=100 H_{*}$ (dash line) and $\beta=500 H_{*}$ (dotted line), with the LISA sensitivity curve.

in Fig. 1 is plotted, along with the spectra for two or three other values of the parameter in question. We can see qualitative outlines of detectability from these figures. For example, Fig. 3 shows that as the temperature of the phase transition increases, detection relies increasingly on the MHD signal.

While these plots give a good qualitative sense of the spectrum dependence on the parameters, we would like to know more precisely which regions of the fourdimensional parameter space of phase transitions result in a gravitational wave spectrum detectable with LISA. The following section gives one useful representation of the detectability regions.

\section{DETECTABLE REGIONS IN PARAMETER SPACE}

The remaining figures show various detectability regions. For the first set of four plots, Figs. 6 to 9, the detectable region in the $\beta / H_{\star}-\alpha$ plane is displayed; each figure is for a different value of the phase transition temperature $T_{*}$, ranging from $0.1 \mathrm{TeV}$ to $100 \mathrm{TeV}$. The larger displayed region is including the MHD inverse cascade from an initial helicity of $\zeta=0.15$, while the smaller region shows the case of no inverse cascade, or equivalently the limit $\zeta=0$. The other parameter, $g_{*}$, is held fixed and only has a weak effect on the results. We designate a power spectrum as detectable if its amplitude comes above the LISA 1-year sensitivity curve at signal-to-noise ratio of 5 at any frequency; more careful detection conditions can also be used but will give the same general results.

Large regions of parameter space are detectable. The natural expectation for $\beta$ is around $100 H_{*}$ [31], with some range around this value for plausible models. For $\beta / H_{\star}$ between 10 and 100, the detection threshold in $\alpha$ tends to be roughly constant, with lower $\alpha$ being detectable as $T_{*}$ increases. If $T_{*}>1 \mathrm{TeV}$, then phase transitions with $\alpha \geq 0.06$ are detectable by LISA. 


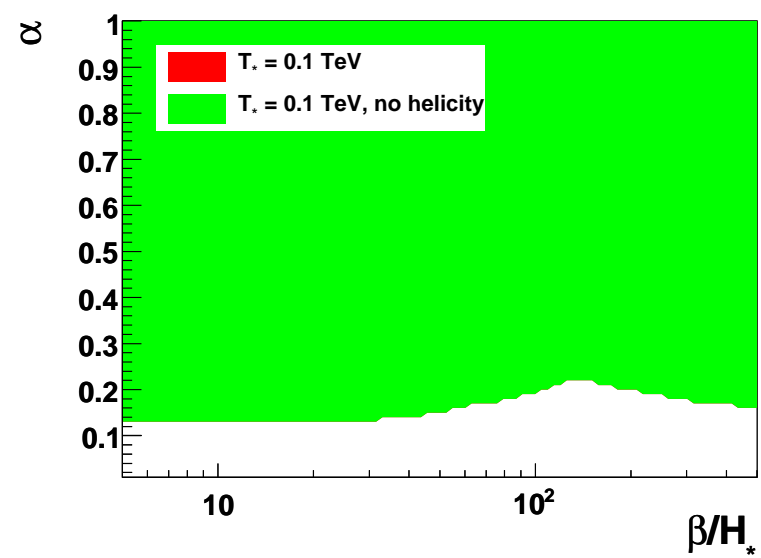

FIG. 6: The $\alpha$ vs. $\beta / H_{\star}$ LISA sensitivity region for a phase transition at a temperature $T_{*}=0.1 \mathrm{TeV}$. The regions for $\zeta_{*}=0$ and $\zeta_{*}=0.15$ coincide at this temperature. A point in parameter space is considered detectable if at any frequency its value of $h_{c}(f)$ is detectable at a signal-noise ratio of 5 in a one-year integration, including the confusion noise from white dwarfs from [43].

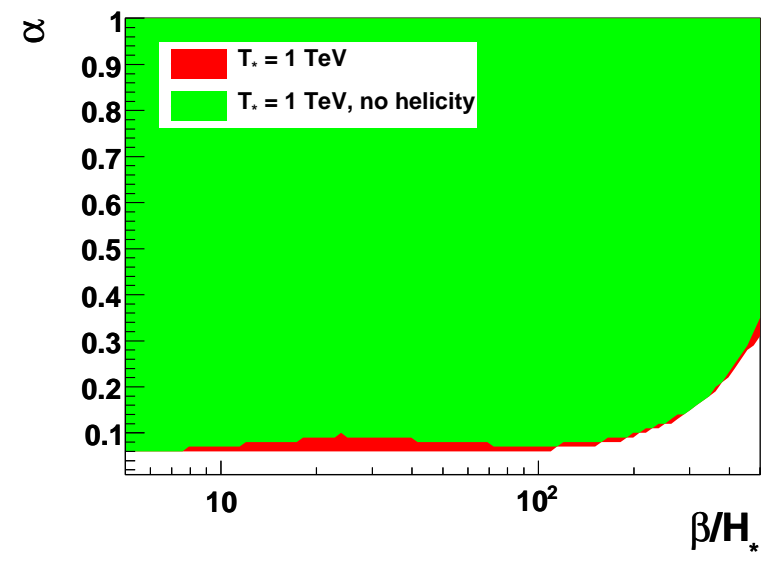

FIG. 7: Same as Fig. 6] except for $T_{*}=1 \mathrm{TeV}$. For $\zeta_{*}=0.15$, a slight additional region along the low- $\alpha$ edge of the $\zeta_{*}=0$ region is detectable.

For temperatures $T_{*} \leq 1 \mathrm{TeV}$, the contribution from the MHD inverse cascade is not important for LISA detectability, due to the experiment's low-frequency cutoff. As $T_{*}$ moves to higher temperatures, the typical frequency, set by $H_{*}$, becomes larger, and the low-frequency peak and tail from the MHD inverse cascade starts to come into the detection window. For $T_{*} \geq 10 \mathrm{TeV}$, the detectability range in $\beta$ is substantially expanded due to the magnetic source, though mostly at comparatively large values of $\alpha$. The spectrum contribution from hydrodynamic turbulence, due to its somewhat higher frequency and amplitude than that from bubble collisions and much higher frequency than the MHD contribution,

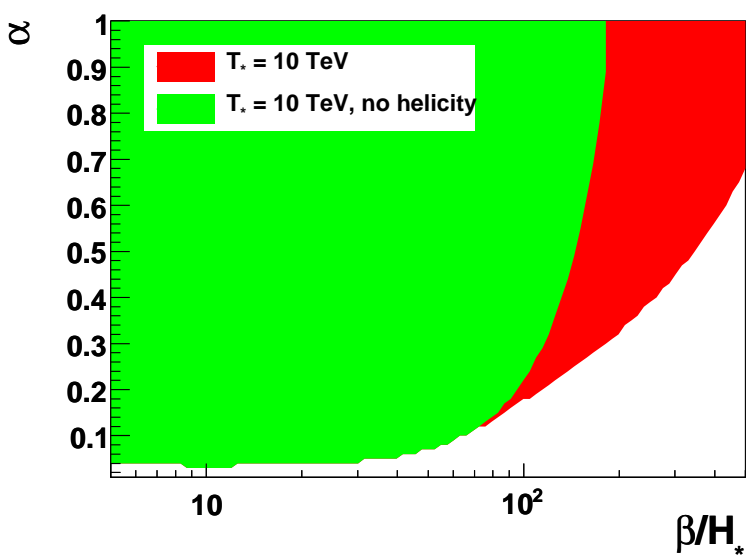

FIG. 8: Same as Fig.6, except for $T_{*}=10 \mathrm{TeV}$. For $\zeta_{*}=0.15$, a substantial extra area on the right side of the $\zeta_{*}=0$ region is detectable.

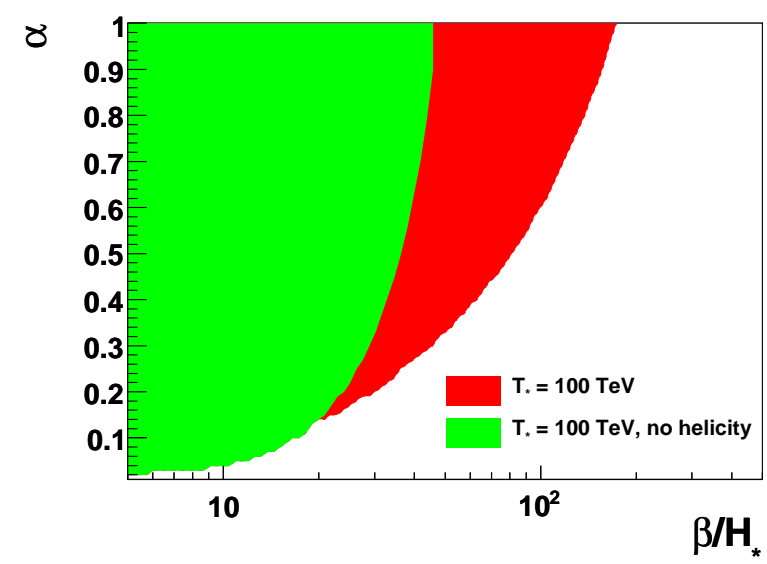

FIG. 9: Same as Fig. 8 except for $T_{*}=100 \mathrm{TeV}$.

dominates most of the detectability for the LISA frequency band.

Figures 10 and 11 show the detectability regions in the $\beta-T_{*}$ plane, for $\alpha=0.1$ and $\alpha=0.5$, respectively. For $\alpha=0.1$, a relatively large region is detectable, while for $\alpha=0.5$, a phase transition at any temperature up to 10 $\mathrm{TeV}$ is detectable for any reasonable value of $\beta$.

These results will be useful for computing future constraints on various high-energy physics theories which give first-order electroweak phase transitions. The fundamental parameters of any theory can be used to construct order parameters and vacuum energies for any phase transition at relevant energy scales (see, e.g., Refs. 22 for many example). These in turn can be used to compute the phenomenological phase transition parameters $\alpha, \beta$, and $T_{*}$ used here, as done in Appendix A of Ref. [4]. The parameter $\zeta_{*}$ likely will require additional astrophysical modelling in situations where there is no well-defined mechanism for generating magnetic fields, but most of 


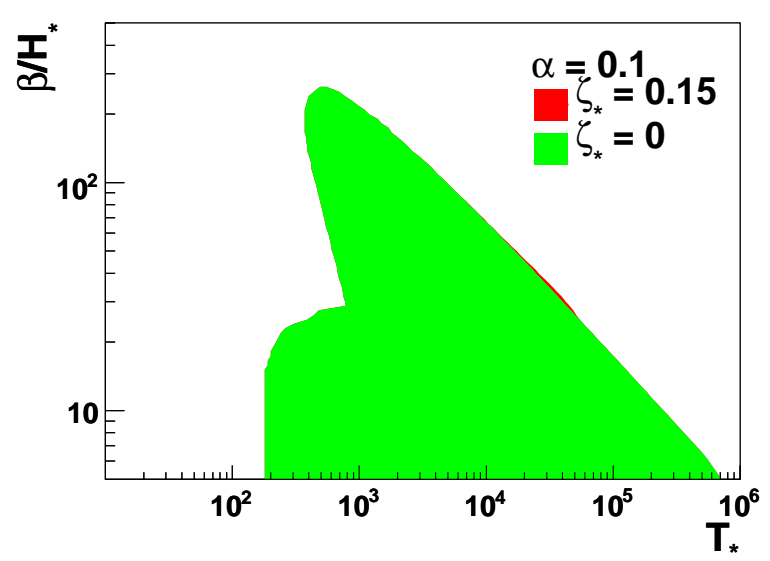

FIG. 10: The $\beta / H_{*}$ vs. $T_{*}$ LISA sensitivity region for a phase transition with vacuum energy $\alpha=0.1$. The regions for $\zeta_{*}=$ 0 and $\zeta_{*}=0.15$ coincide at this temperature. A point in parameter space is considered detectable if at any frequency its value of $h_{c}(f)$ is detectable at a signal-noise ratio of 5 in a one-year integration, including the confusion noise from white dwarfs from [43].

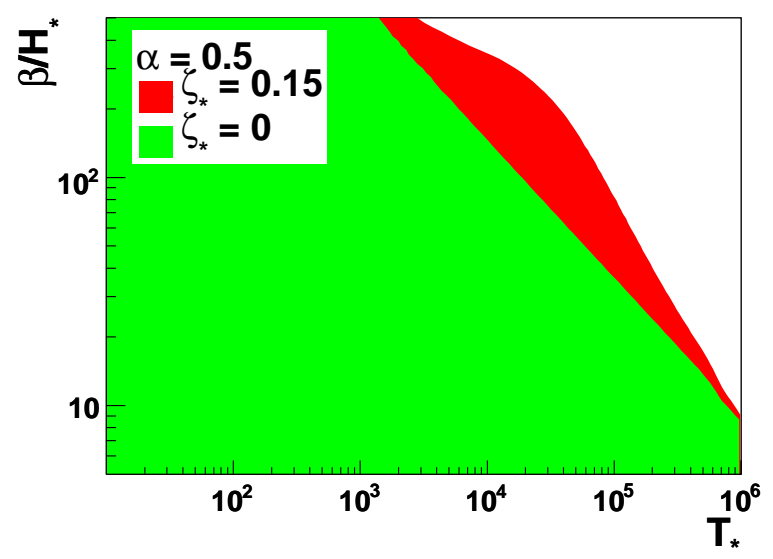

FIG. 11: Same as Fig. 10, except for $\alpha=0.5$.

the parameter-space detectability is independent of this parameter.

We have made several basic physical assumptions: we have assumed that the walls of the bubbles formed in the first order phase transition expand as detonations [34]; that the bubbles remain spherical as they expand; that the resulting bubble collisions source turbulence on the scale of the largest bubbles for a time comparable to the duration of the phase transition; that the turbulence can be modeled as fully-developed Kolmogoroff turbulence; and that initial seed magnetic fields with non-zero helicity will be amplified in an inverse cascade according to a specific MHD turbulence model [39, 40]. While these are all reasonable assumptions and are likely close to the realistic physical situation, they are not guaranteed to be correct. In particular, the assumption that bubbles in a relativistic plasma always expand as detonations deserves further study. The transition from coherent bubble wall expansion to stochastic turbulent behavior needs to be simulated numerically; current computing power is sufficient to model this process with enough resolution to verify the simple assumptions in this work. The standard Kolmogoroff turbulence results apply to non-relativistic fluids, and our direct extension to relativistic plasmas, while likely qualitatively correct, is not based on either direct observation or detailed calculation. Finally, mechanisms for generating seed magnetic fields prior to or during the phase transition are not clearly understood. The enticing prospect of a direct probe of electroweak physics via the stochastic background of gravitational radiation is a strong motivation for further investigation of these interesting questions concerning the fundamental physics of the early universe.

\section{Acknowledgments}

We greatly appreciate useful comments and discussions from L. Campanelli, G. Gabadadze, and B. Ratra. We thank the anonymous referee for spotting a numerical error in an earlier draft of the paper. G.G. and T.K. acknowledge partial support from grant 061000017-9258 from The International Association for the Promotion of Cooperation with Scientists from the Newly Independent States of the Former Soviet Union (INTAS), from grant ST06/4-096 of the Georgian National Science Foundation (GNSF), and from the Associate Member Program of the International Center for Theoretical Physics (ICTP). T.K. and Y.M. have received partial support from grant E-FG02-99ER41093 from the Department of Energy. A.K. has been partly supported from National Science Foundation grant AST-0546035. G.G. acknowledges partial support by the joint Award 06-11 from The Georgian Research and Development Foundation (GRDF) and the U.S. Civilian Research and Development Foundation (CRDF), and grant ST07/4-193 from the Georgian National Science Foundation (GNSF).
[1] L.P. Grishchuk, Sov. Phys. JETP 40, 409 (1975) [Zh. Eksp. Teor. Fiz. 67, 825 (1974)]; A. Starobinsky, Sov. Phys. JETP Lett. 30, 682 (1979) [Pisma Zh. Eksp. Teor. Fiz. 30, 719 (1979)]; V.A. Rubakov, M.V. Sazhin, and
A.V. Veryaskin, Phys. Lett. B 115, 189 (1982); B. Allen, Phys. Rev. D 37, 2078 (1988); B. Ratra, Phys. Rev. D 451913 (1992); M. Giovannini, Phys. Rev. D 60, 123511 (1999). 
[2] S.Y. Khlebnikov and I.I. Tkachev, Phys. Rev. D 56, 653 (1997); R. Easther and E.A. Lim, J. Cosm. Astropart. Phys. 0604, 010 (2006); J. Garcia-Bellido and D.G. Figueroa, Phys. Rev. Lett. 98, 061302 (2007). R. Easther, J. T. Giblin, and E. A. Lim, arXiv:0712.2991 [astro-ph]; J. Garcia-Bellido, D.G. Figueroa and A. Sastre, Phys. Rev. D 77, 043517 (2008); A. Megevand, arXiv:0804.0391 [astro-ph]. .

[3] E. Witten, Phys. Rev. D 30, 272 (1984); C.J. Hogan, Mon. Not. R. Astron. Soc. 218, 629 (1986); M.S. Turner and F. Wilczek, Phys. Rev. Lett. 65, 3080 (1990).

[4] M. Kamionkowski, A. Kosowsky, and M. S. Turner, Phys. Rev. D 49, 2837 (1994).

[5] A. Kosowsky, M.S. Turner, and R. Watkins, Phys. Rev. D 45, 4514 (1992).

[6] A. Kosowsky and M.S. Turner, Phys. Rev. D 47, 4372 (1993); A Kosowsky, M.S. Turner, and R. Watkins, Phys. Rev. Lett. 69, 2026 (1992).

[7] R. Apreda, et al., Class. Quant. Grav. 18, L155 (2001); J.F. Dufaux, et al., Phys. Rev. D 76, 123517 (2007); S.J. Huber and T. Konstandin, arXiv:0709.2091 [hep-ph]; S. J. Huber and T. Konstandin, arXiv:0806.1828 [hep$\mathrm{ph}]$.

[8] T. Vachaspati and A. Vilenkin, Phys. Rev. D 31, 3052 (1985); M.P. Infante and N. Sánchez, Phys. Rev. D 61, 083515 (2000); S. G. Rubin, A.S.Sakharov, M.Yu. Khlopov, J. Exp. Theor. Phys. 91, 921 (2001) [J. Exp. Theor. Phys. 92, 921 (2001)] arXiv:hep-ph/0106187]. .

[9] D.V. Deriagin, et al., Mon. Not. R. Astron. Soc. 229, 357 (1987); R. Durrer, P. Ferreira, and T. Kahniashvili, Phys. Rev. D 61, 043001 (2000); A. Mack, T. Kahniashvili, and A. Kosowsky, Phys. Rev. D 65, 123004 (2002); A. Lewis, Phys. Rev. D. 70, 043011 (2004).

[10] C. Caprini, R. Durrer, and T. Kahniashvili, Phys. Rev. D 69, 063006 (2004).

[11] C. Caprini and R. Durrer, Phys. Rev. D 74, 063521 (2006).

[12] A. Kosowsky, A. Mack, and T. Kahniashvili, Phys. Rev. D 66, 024030 (2002).

[13] A.D. Dolgov, D. Grasso, and A. Nicolis, Phys. Rev. D 66, 103505 (2002).

[14] T. Kahniashvili, G. Gogoberidze, and B. Ratra, Phys. Rev. Lett. 95, 151301 (2005).

[15] G. Gogoberidze, T. Kahniashvili, and A. Kosowsky, Phys. Rev. D 76, 083002 (2007).

[16] http://lisa.nasa.gov/

[17] R. Apreda et al., Nucl. Phys. B 631, 342 (2002).

[18] A. Nicolis, Class. Quant. Grav. 21, L27 (2004).

[19] C. Grojean and G. Servant, Phys. Rev. D 75043507 (2007).

[20] C. Delaunay, C. Grojean and J. D. Wells, J. High Energy Phys. 0804, 029 (2008);

[21] K. Kajantie, M. Laine, K. Rummukainen, and M. Shaposhnikov, Phys. Rev. Lett. 77, 2887 (1996); M. Gurtler, E.M. Ilgenfritz, and A. Schiller, Phys. Rev. D 56, 3888 (1997).

[22] G.W. Anderson and L.J. Hall, Phys. Rev. D 45, 2685 (1992); M. Laine and K. Rummukainen, Phys. Rev. Lett. 80, 5259 (1998); S.J. Huber and M.G. Schmidt, Eur. Phys. J. C10, 473 (1999); S.W. Ham and S.K. Oh, Phys.
Rev. D 70, 093007 (2004); C. Grojean, G. Servant, and J. Wells, Phys. Rev. D 71, 036001 (2005); S. Huber et al., Nuc. Phys. B757, 172 (2006); L. Randall and G. Servant, J. High Energy Phys. 0705, 054 (2007).

[23] J. Cornwall, Phys. Rev. D 56, 6146 (1997); M. Giovannini and M. E. Shaposhnikov, Phys. Rev. D 57, 2186 (1998); G.B. Field and S.M. Carroll, Phys. Rev. D 62, 103008 (2000); T. Vachaspati, Phys. Rev. Lett. 87, 251302 (2001); G. Sigl, Phys. Rev. D 66, 123002 (2002); K. Subramanian and A. Brandenburg, Phys. Rev. Lett. 93, 205001 (2004); L. Campanelli and M. Giannotti, Phys. Rev. D 72, 123001 (2005); V.B. Semikoz and D.D. Sokoloff, Astron. Astrophys. 413, L53 (2005); A. Diaz-Gil, J. Garcia-Bellido, M. Garcia-Perez and A. Gonzalez-Arroyo, arXiv:0712.4263 [hep-ph]; L. Campanelli, arXiv:0805.0575 [astro-ph].

[24] T. Stevens et al., Phys. Rev. D 77, 3501 (2008).

[25] T. Kahniashvili, G. Gogoberidze, and B. Ratra, arXiv:0802.35245 [astro-ph], Phys. Rev. Lett., in press

[26] N. Seto, Phys. Rev. Lett. 97, 151101 (2006).

[27] M. Maggiore, Phys. Rep. 331, 28 (2000).

[28] S. Weinberg, Gravitation and Cosmology (Wiley \& Sons, New York, 1972).

[29] C. Delaunay, C. Grojean and G. Servant, AIP Conf. Proc. 903, 24 (2007).

[30] C. Caprini, R. Durrer, and G. Servant, arXiv:0711.2593 [astro-ph].

[31] M.S. Turner, E.J. Weinberg, and L.M. Widrow, Phys. Rev. D 46, 2384 (1992).

[32] S. Coleman, Phys. Rev. D 15, 2929 (1977); C.G. Callan and S. Coleman, Phys. Rev. D 16, 1762 (1977).

[33] P.J. Steinhardt, Phys. Rev. D 25, 2082 (1982).

[34] M. Kamionkowski and K. Freese, Phys. Rev. Lett. 69, 2743 (1992).

[35] M.E. Goldstein, Aeroacoustics (McGraw-Hill, New York, 1976).

[36] D. Biskamp, Magnetohydrodynamic Turbulence (Cambridge University, Cambridge, 2003); M.K. Verma, Phys. Rep. 401, 229 (2004).

[37] A.S. Monin and A.M. Yaglom, Statistical Fluid Mechanics (MIT Press, Cambridge, MA, 1975).

[38] R.H. Kraichnan, Phys. Fluids 7, 1163 (1964).

[39] D. Biskamp and W.C. Mueller, Phys. Rev. Lett. 83, 2195 (1999); Phys. Plasma 7, 4889 (2000).

[40] M. Christensson, M. Hindmarsh, and A. Brandenburg, Phys. Rev. E 64, 056405 (2001); Astron. Nachrichten 326, 393 (2005).

[41] R. Banerjee and K. Jedamzik, Phys. Rev. D 70, 123003 (2004); L. Campanelli, Phys. Rev. Lett. 98, 251302 (2007).

[42] T. Kahniashvili, L. Campanelli, G. Gogoberidze, and B. Ratra, in preparation

[43] S.L. Larsen, W.A. Hiscock, and R.W. Hellings, Phys. Rev. D 62, 062001 (2000); N. Cornish, Phys. Rev. D 65, 022004 (2001); For a LISA sensitivity curve calculator used here, see http://www.srl.caltech.edu/ shane/sensitivity/

[44] P.L. Bender and D. Hils, Cl. Quant. Grav. 14, 1439 (1997). 